\title{
A philosophical look at the discovery the Higgs boson
}

\author{
Richard Dawid ${ }^{1}$
}

Published online: 18 October 2016

(C) Springer Science+Business Media Dordrecht 2016

\section{Contents}

The missing piece of the puzzle: the discovery of the Higgs boson Allan Franklin

Autopsy of measurements with the ATLAS detector at the LHC Pierre-Hugues Beauchemin

Experimenter's regress argument, empiricism, and the calibration of the large hadron collider

Slobodan Perovic

A case study in experimental exploration: exploratory data selection at the Large Hadron Collider

Koray Karaca

Pragmatic warrant for frequentist statistical practice: the case of high energy physics

Kent Staley

Bayesian perspectives on the discovery of the Higgs particle

Richard Dawid

The Jeffreys-Lindley paradox and discovery criteria in high energy physics Robert D. Cousins

Richard Dawid

richard.dawid@philosophy.su.se

1 Stockholm, Sweden 


\section{The variety of explanations in the Higgs sector}

\section{Michael Stöltzner}

\section{The Higgs discovery as a diagnostic causal inference}

Adrian Wüthrich

\section{Higgs naturalness and the scalar boson proliferation instability problem} James D. Wells

The discovery of the Higgs particle in Summer 2012 marked a milestone in high energy physics. It concluded the search for standard model particles that had been a main goal of experimentation in high energy physics since the early 1970s. It may also lead towards indications for new physics and therefore could emerge as the first step beyond standard model physics. This crucial point in the evolution of experimental high energy physics offers a fitting basis for philosophical reflections on the empirical status of high energy physics.

Recent decades have brought about considerable changes in the layout and character of collider experiments. In a number of ways, empirical testing in contemporary high energy physics differs from the classical understanding of experimental testing. New conceptual issues have emerged that are philosophically significant and must be of concern for a philosophy of physics that aims at being at the height of its time. Those issues are intensely discussed among experimental high energy physicists themselves, which offers a good opportunity for interaction between the philosophy of physics and one of the leading fields of physical research. Moreover, those issues can also have repercussions for the general philosophy of science by raising substantial philosophical questions with respect to the relation between theory and experiment, the epistemic status of theoretical statements and the notions of empirical confirmation and discovery.

The present special issue focuses on empirical and epistemic aspects of the Higgs search and the Higgs discovery. The contributors to the volume, three physicists and seven philosophers of physics, approach this topic from a variety of angles.

The first text by Alan Franklin provides an overview of the experiments that led to the Higgs discovery. Franklin describes the setup of the CMS experiment, including the systems of data selection, the process of reconstructing particles' trajectories and dynamics, and the specific strategies of identifying events potentially containing Higgs particle exchange. He then states the results that established the discovery of a Higgslike particle for both LHC experiments, ATLAS and CMS.

The following three articles circle around the role of theory in setting up and carrying out HEP experiments. The issue of theory-ladenness is of particular significance in the theoretically charged and technically complex context of high energy physics experimentation.

Pierre-Hugues Beauchemin presents an analysis of the way experiments are carried out at the LHC, focusing on various forms in which theory-ladenness enters. He starts with the transformation of raw data into reconstructed events, looks at the mechanism of event selection and the problems it raises for the validation of measurements, and discusses "unfolding", which denotes the method of disentangling effects of the specific measurement process from universal characteristics of a given 
scattering process. Finally, he analyses "systematic uncertainties", the quantification of doubt about a measurement result due to possible conceptual issues. Beauchemin points out that the demonstration of the limited relevance of conceptual uncertainty for data analysis is of substantial importance for understanding the robustness of measurements. This, he concludes, can work against the threat of circularity and provides the basis for justifying claims of objectivity with respect to empirical statements despite the theory ladenness of the experimental process.

Slobodan Perovic reconsiders the debate between Harry Collins and Alan Franklin on what Collins calls the "experimenters' regress". According to Collins, the mutual dependence between the calibration and the success of an experiment turns the final decision on the success of calibration into a sociological issue. Franklin rejects this conclusion based, among others, on the point that calibration mostly relies on the success of other kinds of measurements than the ones to be carried out in the given experiment. Perovic looks closely into the case of Higgs measurements and concludes that one crucial form of calibration, in situ calibration, goes on throughout the experiment itself and therefore is not accomodated by Franklin's view. Still, Perovic argues that this does not decide the issue of the experimenters' regress in Collin's favour since the complex mechanism that relates calibration and experiment at the LHC is not accounted for in Collins' analysis either.

Koray Karaca discusses the issue of experimental exploration in the context of experimental high energy physics. Experimental exploration was introduced by Friedrich Steinle as a mode of experimentation that was not guided by testing a specific theory but by searching for new phenomena in a certain regime without any clear theoretical preconceptions. Karaca argues that, though the concept of empirical exploration in its original form is not applicable in the highly theory driven context of today's high energy physics, it is applicable in the generalized form of what Karaca calls an 'exploratory procedure'. An experiment amounts to an exploratory procedure if it is used for extending the scope of an experimental inquiry beyond what is suggested by the present theoretical status quo. Karaca argues that this concept is particularly helpful for analyzing the choice of data selection mechanisms ("triggers") in high energy physics.

The next three papers deal with the issue of statistical data analysis and the role of frequentist and Bayesian perspectives in that context.

Kent Staley looks at the pragmatic warrant for frequentist methods in high energy physics data analysis. Experimental results in high energy physics are predominantly analyzed by frequentist methods that aim at extracting local and global p-values from the data. Bayesian methods that extract probabilities of hypotheses from the data based on specified prior probabilities are confined to an auxiliary role in specific contexts. Staley argues that the dominant role of frequentist methodology is pragmatically rather than epistemically warranted: the frequentist method is well equipped for conveying pragmatic aspects of the scientific process like the consequences of false announcements. For example, choosing a specific significance criterion for a discovery is motivating by a bundle of considerations. The risk of making a false announcement is among them and plays a role in keeping significance criteria high in high energy physics. 
Richard Dawid views data analysis in high energy physics from a Bayesian perspective. He distinguishes between contexts in high energy physics where Bayesian methods are helpful in statistical data analysis itself and contexts where frequentist data analysis is well established and works well for the physicist but a Bayesian point of view can help develop a broader understanding of the research process. While the former scenarios are usually characterized by the search for generic priors, in the latter scenarios the very point of using a Bayesian perspective is to account for the role of strong commitments in favor of or against the theories to be tested. He exemplifies the second kind of scenario by discussing the role of the look elsewhere effect in the analysis of the Higgs data.

Bob Cousins discusses the merits and problems of the use of Bayesian methods in high energy physics data analysis, using the Jeffreys Lindley paradox as a point of departure. The Jeffreys Lindley paradox denotes the observation that, under specific circumstances, frequentist data analysis can lead to conclusions that are at variance with conclusions extracted from the same data by Bayesian methods. Specifically, a null hypothesis may be rejected based on frequentist methods but preferred based on Bayesian data analysis with generic priors. Cousins shows that high energy physics experiments do create the kinds of scenarios where the Jeffreys Lindley paradox becomes relevant and demonstrates that standard strategies aimed at establishing the irrelevance of the paradox in scientific praxis fail. He then proceeds to discuss a number of issues that arise when deploying Bayesian methods in high energy physics. He concludes that the paradox indicates a core conceptual problem related to cross-calibration of different experiments.

The final three texts deal with the question as to which kinds of inferences can be drawn from the Higgs discovery.

Michael Stöltzner investigates the different forms of the notion of explanation that are applicable to Higgs physics. Stöltzner looks at the five forms of explanation distinguished by James Woodward and finds that all of them can be identified in the context of Higgs physics. He points to the complex relation between the explanatory role of the formalized theory on the one hand and the explanatory role of narratives attached to it (e.g. in the context of "explaining" spontaneous symmetry breaking) on the other. He looks at the various explanatory roles of models that reach beyond the standard model. Further, he emphasizes the connection between levels of explanation and levels of mathematical rigor as exemplified by the different points of view on spontaneous symmetry breaking in high energy physics and axiomatic field theory.

Adrian Wüthrich investigates whether the discovery of the Higgs particle can be understood as a form of causal inference. He argues that particle physics provides paradigm cases of inferences to the existence of unobservable entities based on causal reasoning. He interprets the data analysis leading up to the Higgs discovery in terms of a process of excluding alternative causes of observations. The soundness of this process of 'diagnostic causal inference' faces two interrelated threats: there may be unconceived alternatives to the identified cause and the characteristics of the object conjectured as a cause cannot be exhaustively specified in advance. Wüthrich argues that these threats are controlled in high energy physics by carefully defining the levels of specificity addressed by a given experiment. The characterization of the inferred particle remains unspecific in all respects not univocally determined by the data. 
In the final article, James Wells looks beyond the standard model. Unlike in the case of particles with spin, perturbative corrections to the Higgs mass give contributions of the order mass squared of particles included in those corrections. If such contributions arose in an unconstrained way, implausible finetuning in summing up those huge contributions would be required to generate the measured low Higgs mass. Now, it is generally assumed that there exist particles with masses close to the grand unified scale or the Planck scale, that is, many orders of magnitude above the Higgs mass. Wells argues that, when thinking about specific scenarios, the described finetuning problem in effect boils down to the contributions from very massive fundamental scalars. This is to be taken very seriously, however, since assuming that the discovered Higgs particle is the only fundamental scalar in nature seems unsatisfactory. Low energy supersymmetry looks like the most promising way to cancel quadratic contributions and therefore avoid finetuning even in the presence of highly massive scalars. 